\title{
NINO Y DWORKIN SOBRE LOS CONCEPTOS DE DERECHO
}

\author{
J.J. MoReso \\ Universitat Pompeu Fabra
}

\section{Resumen}

Algunos de los más relevantes filósofos del derecho de los últimos años, como Carlos S. Nino y Ronald Dworkin, han defendido que hay una pluralidad de conceptos de Derecho. Scott Shapiro ha sostenido una posición especialmente relevante acerca de ello: la palabra 'Derecho' es sistemáticamente ambigua, pues a veces designa un conjunto de normas y otras veces una organización social. Esta es precisamente la tesis criticada en el trabajo. Se argumenta, basándose en determinada literatura filosófica acerca de los conceptos, que los conceptos de objetos sociales de carácter intencional (como es el derecho, pero también las obras literarias por ejemplo) contienen una dimensión social y una dimensión significativa, normativa en el caso del derecho. El mismo concepto de Derecho expresa una dimensión social y una normativa imbricadas entre sí.

PALABRAS CLAVE: Carlos S. Nino; Ronald Dworkin; concepto de derecho; pluralismo conceptual.

\begin{abstract}
Some of the most relevant legal philosophers in the last decades, as Carlos S. Nino and Ronald Dworkin, have defended the idea that there is a plurality of concepts of law. Scott Shapiro has sustained this account in a particularly relevant way: the word 'law' displays a ubiquitous ambiguity, sometimes designates a set of norms and other a social organization. This is precisely the thesis which is criticized in the paper. It is argued, from a certain philosophical literature about the concepts, that concepts of social objects of intentional character (as it is the case for law, but also for literary works for example) contain a social dimension and a meaning dimension, normative in the case of the law. That is to say, the concept of law expresses a social dimension and a normative dimension intertwined.
\end{abstract}

KEY WORDS: Carlos S. Nino; Ronald Dworkin; Concept of Law; Conceptual Pluralism.

Cuando yo escribo algo, tengo la sensación de que ese algo preexiste. Parto de un concepto general; sé más o menos el principio y el fin, y luego voy descubriendo las partes intermedias; pero no tengo la sensación de inventarlas, no tengo la sensación de que dependan de mi arbitrio; las cosas son así. Son así, pero están escondidas y mi deber de poeta es encontrarlas. Jorge Luis Borges, Siete noches 
Yesterday,

All my troubles seemed so far away, Now it looks as though they're here to stay

Oh I believe in yesterday.

Lennon y McCartney, Yesterday

\section{Sobre la pluralidad de los conceptos de derecho}

A pesar de que la obra con la que se inicia el debate contemporáneo en teoría jurídica, principalmente en el mundo anglosajón, es The Concept of Law (1961/2012) de H. L. A. Hart y que Hart parecía suponer que es posible moldear un concepto de derecho, entendido como sistema jurídico (legal system, municipal law como dice muchas veces en el libro) capaz de capturar los rasgos sobresalientes de esta práctica social diferenciándola de otros fenómenos cercanos, pero distintos, como la coerción y la moralidad (así comienza el prefacio del libro, Hart 1961/2012, p. vi), en los últimos años prominentes filósofos del derecho han sostenido que no hay un único concepto DERECHO. ${ }^{1}$

Han sostenido que hay, por el contrario, una pluralidad de conceptos DERECHO que significan cosas diversas según los contextos en los que son empleados. No en el sentido obvio de que en castellano la palabra 'derecho' es ambigua: no significa lo mismo dicha expresión en las siguientes oraciones 'El derecho español prohíbe la pena de muerte', 'Tengo derecho a que me prestes atención' o 'Ve derecho hasta el próximo

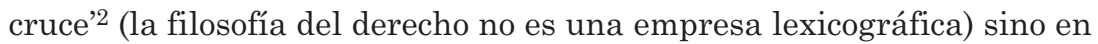
el sentido de que cuando la palabra 'Derecho' se refiere al sistema jurídico español, norteamericano, argentino o al sistema jurídico de la Antigua Roma, hay que tener en cuenta que no connota un único concepto DERECHO, sino una pluralidad de ellos.

${ }^{1}$ Se seguirá aquí la convención habitual ahora en la filosofía analítica de usar una palabra con todas las letras en mayúscula para referirse al concepto que la palabra connota. Así: POEMA se refiere al concepto de poema, y no a su referencia, ni a la expresión lingüística, ni tampoco a la propiedad de ser un poema o al significado de 'poema' (cuestiones estas últimas claramente relacionadas con el concepto POEMA, pero acerca de las cuales hay controversia en filosofía).

${ }^{2}$ Una ambigüedad, por cierto, algo diferente a la que la expresión 'Law' tiene en inglés, dado que en inglés se distingue entre 'law' y 'right' y, por lo tanto, 'Law' no es pasible de algunas de las ambigüedades de 'derecho' (y de las expresiones equivalentes en las lenguas latinas ('droit', 'diritto') y en alemán ('Recht'). Pero es pasible de otras ambigüedades porque hay laws of nature (Boyle and Mariotte's law) y laws of logic (law of excluded middle), por ejemplo. Véase Raz (1994b, p. 198), Shapiro (2011, pp. 7-8) y Gardner (2012b, p. 177). 
El primer autor que sostuvo claramente esta posición, hasta donde se me alcanza, fue Carlos Santiago Nino (1985b, 1994). Pero después ha sido asumida por diversos autores. En este trabajo analizaré también la posición de Ronald Dworkin (2006a, 2006b) y comentaré una forma realmente instructiva de comprender la pluralidad de conceptos de derecho, tal como la desarrolla Scott J. Shapiro (2011). Presentaré la posición de estos autores y, también, las dudas que suscita, para terminar defendiendo que una adecuada concepción de los conceptos no tiene por qué conducirnos a esta conclusión.

Nino (1994, p. 32) expresaba de este modo contundente su posición:

Estas consideraciones sugieren que respecto del derecho, como sin duda en relación con muchos otros conceptos, lo apropiado sería adoptar una posición convencionalista. Según esta posición, el concepto de derecho surge de estipulaciones y prácticas que tienen en cuenta las necesidades del discurso en el que ese concepto se emplea. La consecuencia inmediata de esta posición es la admisión de que puede haber una pluralidad de conceptos de derecho, ya que las necesidades del discurso pueden variar en y con el discurso. La percepción de que hay diversos discursos relacionados con el derecho, con funciones y puntos de vista muy diferentes hace pensar que en ellos se emplean nociones de derecho diferentes, aunque es posible [...] que estén relacionadas entre sí.

Nino (1985b, pp. 194-5), por otra parte, consideraba que una posición pluralista respecto a los conceptos DERECHO, tendría la virtud de aclarar y disolver algunas de las controversias más importantes en la teoría jurídica contemporánea:

Es como si subsistiera un último reparo a abandonar un enfoque absolutista respecto de la noción básica del esquema conceptual que debe reconstruirse, y cuya singularidad permitiría dar unidad a todo el sistema de conceptos [...] Creo que el panorama de la filosofía del derecho se despejaría considerablemente y cambiaría en forma substancial la naturaleza de los problemas a enfrontar ... si admitimos que la 'clientela' de la filosofía jurídica no está constituida por un solo discurso acerca del derecho (sea el de la ciencia jurídica o el de la sociología, o el de la administración de justicia, según las preferencias del filósofo), que se satisface mediante la reconstrucción de un aparato conceptual unitario, sino por una pluralidad de puntos de vista frente al derecho que requieren de distintos sistemas de conceptos. 
Bien, pues estas son las tesis que deseo cuestionar en esta contribución ahora que se han cumplido los veinte años desde que nos dejó, lo que lamentablemente nos impide conocer sus agudas réplicas, con las que siempre nos impresionaba y, por qué no reconocerlo, a menudo nos abrumaba con su inteligencia extrema. Y lo haré del siguiente modo: en el segundo epígrafe se presentan los ocho conceptos de derecho que Nino detectaba en la literatura teórico-jurídica. En el tercero, se presentarán los cuatro conceptos que Dworkin distingue. Con las ideas de Shapiro, en el cuarto epígrafe, se tratará de comprender la tesis de la pluralidad de conceptos de derecho como resultado de la ambigüedad ubicua de las expresiones 'derecho' y 'sistema jurídico', con la intención de mostrar que la idea de ambigüedad no es adecuada para reconstruir los conceptos referidos a prácticas humanas de carácter intencional. En el quinto epígrafe, y a partir de las conclusiones obtenidas en el anterior, se avanzarán algunas razones para dudar de la adecuación de los enfoques de Nino y Dworkin en este punto. En el sexto, se introducirá una visión general de la naturaleza y la estructura de los conceptos en la filosofía analítica contemporánea, con especial énfasis en su estructura para defender una concepción de los conceptos que combine la teoría de los prototipos con el enfoque teórico capaz de iluminar nuestra reconstrucción del concepto DERECHO. Y con ello se llegará a la conclusión de que es el mismo y único concepto DERECHO el que exhibe tanto una dimensión social como una dimensión significativa imbricada con lo normativo. Ser más consciente de ello arroja dudas sobre la tesis con arreglo a la cual las discrepancias acerca de la naturaleza del derecho son solo disputas de palabras y permite atender a cómo estas discusiones presuponen desacuerdos filosóficos importantes. El debate que al respecto mantuvo Nino con Genaro R. Carrió cerrará el trabajo como muestra de este último punto.

\section{Los ocho conceptos de derecho en Nino}

Apoyado en este convencionalismo conceptual, Nino defendió que hay conceptos descriptivos de derecho, conceptos normativos y conceptos mixtos (1994, pp. 35-40). En concreto, son los siguientes:

En primer lugar, un concepto descriptivo realista de derecho $\left(\mathrm{DERECHO}_{1}\right)$, con arreglo al cual este concepto se refiere a los estándares que son o probablemente serán aceptados por los órganos de aplicación como justificación de sus decisiones. Lo atribuye a Alf Ross. ${ }^{3}$

${ }^{3}$ Cf. por ejemplo Ross (1958). 
En segundo lugar, un concepto descriptivo sistemático de derecho $\left(\right.$ DERECHO $\mathrm{CH}_{2}$ ), conforme al cual el concepto denota aquellas pautas identificadas como en el caso anterior y sus consecuencias lógicas. Lo atribuye a Carlos E. Alchourrón y Eugenio Bulygin. ${ }^{4}$

En tercer lugar, el último de los conceptos descriptivos $\left(\right.$ DERECHO ${ }_{3}$ ), que restringe el primero, dado que se limita a identificar los estándares reconocidos por los órganos de aplicación que han sido prescritos por cierta autoridad o provienen de cierta fuente. Y así excluyen las pautas, como los principios dworkinianos, que no proceden de fuente social alguna. Obviamente es Joseph Raz su más conspicuo defensor. ${ }^{5}$

Y, a continuación, Nino nos propone tres conceptos normativos de derecho y dos mixtos. Son los siguientes:

En cuarto lugar, un concepto de lege ferenda $\left(\right.$ DERECHO $\left._{4}\right)$, según el cual el derecho está integrado por todas aquellas pautas que deben ser reconocidas en el ejercicio del monopolio de la fuerza de los poderes públicos. Reconoce que este concepto puede afinarse en otros dos.

En quinto lugar, un concepto normativo judicial amplio $\left(\right.$ DERECHO $\left._{5}\right)$, que se refiere a las pautas que deben ser reconocidas por los órganos de aplicación en la decisión de los casos concretos. Puede haber pautas, añade, que aunque deben ser aceptadas por los legisladores no deben ser aplicadas por los jueces.

En sexto lugar, un concepto normativo judicial restringido $\left(\right.$ DERECHO $\left._{6}\right)$, que restringe las pautas referidas en el supuesto anterior a aquellas que han sido prescritas por determinada autoridad legítima.

En séptimo lugar, el primer concepto mixto de derecho, $\left(\right.$ DERECHO $\left._{7}\right)$, un concepto que identifica las pautas que los jueces de hecho aceptan y aplican y también aquellos que deben ser reconocidos como la mejor justificación de su práctica de aplicación. Como es obvio, lo atribuye a Ronald Dworkin. ${ }^{6}$

Por último, el octavo concepto de derecho, un concepto normativo hipotético de derecho, $\left(\mathrm{DERECHO}_{8}\right)$, un conjunto de pautas solo hipotéticamente válidas y que, por lo tanto, son de hipotética obligatoria aplicación para los órganos de aplicación. Una concepción que, sin duda, tiene sabor kelseniano. ${ }^{7}$

De todo ello Nino concluye (1994, pp. 41-42) que muchas de las tradicionales disputas iusfilosóficas son espúreas: así, por ejemplo, la

${ }^{4}$ Cf. Alchourrón,y Bulygin (1971) y (1981).

${ }^{5}$ Por ejemplo Raz (1994a).

${ }^{6}$ Cf. Dworkin (1977) y (1986).

${ }^{7}$ Puede verse Kelsen (1960). 
discusión sobre las relaciones entre el derecho y la moralidad. La respuesta es simple: para algunos conceptos de derecho, los conceptos normativos, hay necesariamente esta conexión; para otros, en cambio, como los descriptivos, tal conexión es meramente contingente.

\section{Dworkin: la concepción interpretativa del concepto de derecho}

Dworkin (2006a, 2006b, pp. 2-5, 223-225) distingue cuatro conceptos de derecho. El primero, y fundamental para él, es el concepto doctrinal de derecho $\left(\mathrm{DERECHO}_{1}\right)$ que se encuentra usado cuando decimos cosas como 'el derecho español reconoce los matrimonios entre personas del mismo sexo' o 'el derecho español prohíbe circular a más de $120 \mathrm{~km} / \mathrm{h}$ por las autopistas'. En la concepción de Dworkin las dos anteriores oraciones expresan proposiciones jurídicas (propositions of law) y es controvertido de qué dependen sus condiciones de verdad. Es claro que, a menudo, dependen de lo que han establecido las autoridades legislativas, pero tal vez también los precedentes judiciales y, según Dworkin, los principios morales que mejor dan cuenta de la práctica jurídica en cuestión.

El segundo es el concepto sociológico de derecho, $\left(\mathrm{DERECHO}_{2}\right)$, con el cual nos referimos a una particular estructura social de carácter institucional. Con este concepto podemos afirmar, por ejemplo, que el derecho sirve para reducir las agresiones contra la vida y la integridad física, o que los romanos desarrollaron una sofisticada y compleja forma de derecho (Dworkin 1996b, p. 223).

En tercer lugar, tenemos el concepto taxonómico de derecho, $\left(\right.$ DERECHO$\left._{3}\right)$, en virtud del cual distinguimos aquellas pautas que integran un sistema jurídico de otras pautas (morales, estéticas, aritméticas, etc.) que no forman parte del derecho. Claro que ello es compatible con que las condiciones de verdad de algunas proposiciones jurídicas dependan de algunas de estas pautas externas. Para identificar la proposición que establece que el derecho español prohíbe circular por las autopistas a más de $120 \mathrm{~km} / \mathrm{h}$ es necesario usar las reglas del sistema métrico decimal, pero nadie diría que el sistema métrico decimal es parte del derecho.

En cuarto y último lugar, tenemos el concepto aspiracional (aspirational) de derecho, $\left(\mathrm{DERECHO}_{4}\right)$, que establece el ideal de que el derecho se adecúe a los estándares del Estado de derecho (la rule of law), aunque a veces no alcance este objetivo.

$\mathrm{Al}$ menos en un lugar (2006, p. 2, n. 1 en p. 263) Dworkin se 
interroga acerca de si no podría replicarse a sus distinciones con el argumento de que solo existe un concepto DERECHO que es usado de modos diversos. Y, aunque acepta que estos conceptos están interrelacionados entre sí, rechaza el argumento porque sostiene que se refieren a realidades distintas. Así, el concepto doctrinal se refiere a proposiciones que pretenden ser normativamente válidas, en cambio el concepto sociológico se refiere a instituciones y a pautas de comportamiento. Volveré enseguida sobre ello.

\section{Las palabras 'derecho' y 'sistema jurídico' son ambiguas: Shapiro}

Shapiro (2011, pp. 4-7) sostiene que tanto 'derecho' como 'sistema jurídico' son palabras ambiguas. Pueden funcionar en el discurso como sustantivos incontables (mass terms), como dicen los lingüistas, por ejemplo 'nieve', 'trigo', 'dinero', y entonces se refieren a una cantidad no contable de normas jurídicas. De un modo semejante a como decimos que hay un exceso de nieve en el jardín, podemos decir que en el derecho español hay un exceso de regulación sobre, por ejemplo, el acceso a las universidades públicas de los estudiantes. Pero 'derecho' y 'sistema jurídico' también puede referirse a una especial organización social. Y entonces decimos cosas como 'el derecho, o el sistema jurídico, disciplina el uso de la fuerza' para referirnos a la organización social integrada por las autoridades legislativas que crean las normas, los jueces y Tribunales que las aplican o la policía que, a menudo, las ejecuta.

Entonces, para Shapiro, hay dos conceptos de derecho, DERECHO y $\mathrm{DERECHO}_{2}$, dado que la expresión 'derecho' es ambigua. Del mismo modo que 'luna' es ambiguo porque puede referirse al satélite de la tierra, LUNA $_{1}$, y también al cristal de un espejo, LUNA ${ }_{2}$. Si Shapiro lleva razón, entonces no hay misterio alguno en la idea de la pluralidad de conceptos de derecho. 'Derecho' cuando se refiere a un sistema jurídico es una palabra ambigua y, por lo tanto, denota realidades diversas y connota al menos más de un concepto.

Sin embargo, ni es claro que esta sea la idea de Nino y de Dworkin, aunque algunas veces parecen suscribirla, ni es claro que esta sea una idea plausible.

Mis dudas al respecto surgen dado que, si Shapiro llevara razón, entonces muchas palabras que se refieren a productos humanos de carácter intencional serían ambiguas: las novelas, las canciones, las conversaciones ordinarias, las obras de arte como los cuadros o las esculturas. Pensemos en la expresión 'la canción Yesterday de los Beatles'. 
De ella podemos decir cosas diversas: que se trata de la canción más escuchada del grupo, que es la canción más veces versionada de la historia, que fue (la letra y la música) creada por Paul McCartney, que dio a los miembros del grupo una cantidad increíble de beneficios. Pero también podemos decir que la canción evoca un desencuentro amoroso y que alcanza su estatus de balada romántica, al menos en parte, gracias al cuarteto de cuerda que acompaña su melodía. ¿Tenemos entonces dos conceptos YESTERDAY, (YESTERDAY 1 y YESTERDAY $\left.{ }_{2}\right)$ ? YESTERDAY $_{1}$ referido a la práctica de crear la canción y reproducirla y YESTERDAY $_{2}$ referido al contenido, el significado de la letra de la canción y la música. Creo que no. Todas las creaciones humanas intencionales tienen esta característica: tienen un contenido que solo puede existir incorporado en un soporte físico que mantiene relaciones de causalidad con otros objetos del mundo. Se trata de la misma canción que consigue emocionarnos con una letra algo naif y adolescente, tal vez por haber hallado la melodía adecuada, y que es abrumadoramente reproducida, causa emociones diversas en multitud de oyentes, fue elaborada por McCartney. Todos los productos humanos intencionales integran un contenido significativo incorporado, como afirma Searle (1983, p. 151), the body takes over. ${ }^{8}$

Esto ocurre, más simplemente, con las emisiones lingüísticas: si yo digo a un amigo 'Lograste que me enfadara', esta expresión tiene un contenido, por ejemplo presupone que el destinatario de la emisión hizo algo que me sentó mal (su dimensión locutiva) y a la vez expresa una queja y causa, supongamos, malestar y arrepentimiento en el destinatario (las dimensiones ilocutiva y perlocutiva).

Pero ello no significa que haya dos conceptos de emisión lingüística. Hay un solo concepto, aunque complejo, que tiene varias dimensiones relacionadas entre sí. El contenido de significado, aquello que la hace intencional, es lo que permite a la emisión tener después los efectos causales en el mundo, porque es proferida por alguien, dirigida a algún otro. Una práctica lingüística es una práctica social significativa, dotada de contenido. Lo mismo ocurre con el concepto de la canción Yesterday, YESTERDAY es un concepto complejo que se refiere a una práctica social significativa. Por cierto, esto no significa que la práctica esté determinada con absoluta precisión. Podemos discrepar en si una versión, por ejemplo con la letra traducida al español, es todavía una instancia de YESTERDAY, o con algún pequeño cambio en la letra o en la melodía. El concepto YESTERDAY, como todos los conceptos que se refieren al mundo, es vago.

${ }^{8}$ Me doy cuenta de la importancia de esta idea de Searle gracias a Celano (ms). 


\section{El concepto DERECHO}

Trataré ahora de aplicar estas ideas al concepto DERECHO (SISTEMA JURÍDICO). Comenzaré con una anécdota instructiva que oí contar una vez a Eugenio Bulygin. El importante profesor español de Derecho Penal, Luis Jiménez de Asúa, que había sido presidente de la Comisión Constitucional encargada de elaborar el proyecto de Constitución de la II República española en 1931, después de la guerra civil se exilió en Buenos Aires (era un destacado miembro del Partido Socialista en España) y llegó a ser profesor de Derecho Penal en la Facultad de Derecho de la Universidad de Buenos Aires. A la vez, algunos de los exiliados españoles mantuvieron la ficción de continuidad de las instituciones republicanas, como modo de ejercer una presión internacional (infructuosa como bien sabemos) contra la dictadura del general Franco. En el exilio, Jiménez de Asúa fue elegido Presidente de las Cortes y, según el artículo 74 de la Constitución de la II República, el Presidente de las Cortes se convertía en Presidente de la República en caso de fallecimiento o incapacidad de este. En el año 1962 murió Diego Martínez Barrio, que era el Presidente de la República en el exilio, y lo sucedió en la presunta presidencia Luis Jiménez de Asúa. Entonces algunos profesores conservadores de la Facultad de Derecho de la Universidad de Buenos Aires, arguyeron que debía ser aplicada una regulación de dicha Universidad que prohibía a los Jefes de Estado de otros países formar parte del claustro de la Universidad y solicitaron que Jiménez de Asúa fuese cesado.

Pues bien, con el concepto doctrinal de Derecho de Dworkin no hay una manera clara de oponerse a esta conclusión. Sin embargo, fue rechazada como una idea mendaz. Todo el mundo sabía que el Jefe del Estado español en 1962 era el general Franco, que había vencido una cruenta guerra civil y había impuesto un régimen dictatorial por medio de las armas y el terror. Jiménez de Asúa podía ser el Jefe del Estado español conforme a la Constitución de la II República, sin embargo el derecho español no se fundaba ya en dicha Constitución y, de acuerdo con las Leyes Fundamentales del franquismo, el Jefe del Estado no era Jiménez de Asúa.

Para alcanzar esta obvia conclusión necesitamos darnos cuenta de que el concepto de derecho español en 1962 se refería a un conjunto de normas en vigor, en virtud de determinados hechos sociales, trágicos en esta ocasión. Que con la victoria de Franco en la guerra civil, los operadores jurídicos en España (los legisladores, los jueces, la policía, los abogados, etc.) identificaban el derecho con las Leyes Fundamentales y 
la legislación creada de acuerdo con ellas. Unos lo hacían por convicción, otros lo hacían solo por miedo. Pero lo hacían de este modo y la comunidad internacional así lo reconocía (a mitad de los años cincuenta España fue aceptada como miembro de Naciones Unidas, por ejemplo). Esto es, la dimensión doctrinal del Derecho depende de su dimensión social. Es imposible establecer lo que el Derecho requiere en una determinada jurisdicción sin una primera identificación de los hechos sociales de los cuales depende la existencia de un determinado sistema jurídico.

Por esta razón, es oportuna la consideración de Gardner (2012a, p. 270) cuando sostiene que la afirmación de Dworkin (2006a, p. 95) de que los conceptos doctrinal y sociológico de derecho están interconectados es un understatement. Sucede que DERECHO dice referencia a una práctica social con un determinado contenido de significado. Nadie diría que el Parlamento o los jueces forman parte del derecho, como nadie diría que McCartney forma parte de la canción Yesterday, pero el concepto de derecho es complejo y presupone la existencia de dichas instituciones. Dicho de un modo hartiano, la dimensión social del derecho es su aspecto externo, que tiene relaciones de causalidad en el mundo, la dimensión doctrinal constituye su aspecto interno, que captura su significado.

¿Qué decir entonces de los otros dos conceptos dworkinianos, el concepto taxonómico y el concepto aspiracional?

Bien, por lo que respecta al concepto taxonómico, Dworkin (2006b, pp. 232-240) lo considera una cuestión intrascendente. Insiste en que aunque a veces los jueces deben recurrir a las reglas de la aritmética, por ejemplo para calcular el monto de una deuda, dichas reglas no integran el derecho y, de un modo más relevante, que cuando el derecho español establece por ejemplo que la capacidad para testar de un extranjero, pongamos un croata, que otorga su testamento en España, debe ser validada siguiendo las reglas del derecho de Croacia, no por ello dichas reglas se incorporan al derecho español. Este es un argumento múltiples veces usado por Joseph Raz (1979, pp. 101-102, 119-120; cf. también Coleman 1998, pp. 404-405). Sin embargo el argumento de Raz, a pesar de lo que dice Dworkin, no es un argumento meramente taxonómico, sino doctrinal. Cuando se trata de las reglas de la artimética o del derecho de Croacia, Raz diría que las normas de la jurisdicción que remiten a otras normas pretenden autoridad y consiguen dirigir el comportamiento de los jueces, excluyendo otras consideraciones. Lo que ocurre en el caso de la moralidad es distinto. La razón por la que Raz (por ejemplo Raz 2004) no admite la incorporación de conceptos y argumentos morales en la identificación del derecho es doctrinal, supone que dicha incorporación privaría al derecho de autoridad, porque dado que los jueces ya están 
sometidos a la moralidad, la moralidad dispone de jurisdicción universal, dichas pautas morales no consiguen desplazar las razones que el juez tiene para tomar su decisión, fracasando así como pautas dotadas de autoridad jurídica. Es decir, son las razones que llevan a moldear el concepto de derecho de Raz, un concepto doctrinal, las que le llevan a la conclusión que Dworkin califica de taxonómica. ${ }^{9}$

Por último en relación con Dworkin, ¿qué decir del concepto aspiracional de derecho? Bien, el concepto aspiracional de derecho es semejante al concepto de derecho ideal, aquel que reúne en modo óptimo las virtudes que, desde un punto de vista valorativo, atribuimos a los sistemas jurídicos. ${ }^{10}$ Ahora bien, de igual manera que el concepto de bicicleta ideal captura algún elemento nuclear de la naturaleza de las bicicletas y está conectado con nuestra noción BICICLETA, pero no sirve para distinguir las bicicletas de lo que no lo son, porque hay muchas bicicletas que son bastante menos que ideales sin dejar de serlo, el concepto aspiracional de derecho no es el concepto DERECHO, aunque pueda iluminar aspectos de él, sino solo una particular instancia genérica de tal concepto.

Veamos ahora lo que ocurre con los ocho conceptos de DERECHO propuestos por Carlos S. Nino. A pesar de la minuciosidad de Nino, creo que los ocho conceptos pueden ser reducidos a tres: un concepto descriptivo de derecho, un concepto normativo de derecho y un concepto mixto. Los tres conceptos descriptivos, como los tres normativos, son solo refinamientos de una sola idea, un solo concepto. Por ejemplo, el hecho de discrepar acerca de si mis creencias incluyen lo que está implícito -implicado- en mis creencias explícitas o no, no es suficiente para decir que tenemos diversos conceptos CREENCIA, se trata solo de que discrepamos acerca de la mejor reconstrucción conceptual de dicha noción. Lo mismo ocurre con los conceptos descriptivos de derecho de Nino. Pero esto no es lo más relevante. Lo más relevante es que, al revés de lo que sucedía con Dworkin, sin atender a la dimensión normativa no es ni siquiera posible delimitar las pautas que vamos a considerar 'derecho'. Cuando los juristas norteamericanos discrepan acerca de si la enmienda octava de su Constitución, que prohíbe los cruel and unusual punishments, excluye o no la pena de muerte, no podemos resolver esta cuestión con un enfoque meramente descriptivo. Si la respuesta dependiera de lo que los Tribunales han hecho hasta ahora, esto es, no

${ }^{9}$ Aunque no comparto la argumentación de Raz (Moreso 2009, ensayo 10) y (2013), este no es el lugar para reproducir esta discusión.

10 Una reciente y perspicua contribución a esta noción en Laporta (2007). 
considerarla inconstitucional, entonces la polémica carecería de sentido. Si la polémica tiene sentido, y creo que la tiene, es porque no es posible determinar lo que la Constitución de los Estados Unidos establece sin atender a lo que presupone, y lo que presupone solo puede identificarse mediante el uso de argumentos valorativos.

Es decir, el concepto DERECHO es un concepto complejo, mixto para decirlo con Nino, que hace referencia a determinadas prácticas sociales en un contexto necesariamente evaluativo. Esto comporta que el concepto DERECHO es un concepto controvertido, que hay una disputa genuina sobre cuál sea la naturaleza del derecho. Para hacer lugar a dicha posibilidad es aconsejable detenerse un poco en la naturaleza de los conceptos.

\section{El concepto de concepto}

Trataré aquí de presentar el panorama en la filosofía actual acerca de dos cuestiones centrales: i) la naturaleza de los conceptos y ii) la estructura de los conceptos (siguiendo de cerca Margolis y Laurence 2014).

Acerca de la ontología de los conceptos hay tres opciones sobresalientes: los conceptos son o bien representaciones mentales, o bien aptitudes o bien entidades abstractas (sentidos fregeanos).

La primera posición, los conceptos como representaciones mentales, procede del empirismo clásico (era la concepción, como es sabido, de John Locke y David Hume). Para esta concepción los conceptos son entidades psicológicas que pueblan nuestras mentes formadas a partir de nuestra percepción sensorial de los objetos del mundo. Es la concepción predominante de los filósofos cercanos a los desarrollos de la ciencia cognitiva (véase, por todos, Pinker 1994).

La segunda posición surge como resultado del escepticismo wittgensteniano (Wittgenstein 1953) acerca de la utilidad de las representaciones mentales en filosofía. Para esta concepción, los conceptos son aptitudes de los sujetos cognitivos que les permiten discriminar diversos objetos y sucesos en el mundo. El concepto ARBUSTO consiste en la aptitud de distinguir aquellas plantas que son arbustos de los demás objetos del mundo.

Para el tercer enfoque los conceptos son entidades abstractas que integran y componen las proposiciones. Son las entidades que median entre el pensamiento y el mundo, de un lado, y la realidad a la que se refieren, su referencia, de otro (véase por ejemplo Peacocke 1992).

Sin embargo, creo que no hace falta, para nuestro objetivo centrado en el concepto DERECHO, involucrarse en esta polémica. Tal vez sea posible generar alguna posición ecléctica, en la cual a nuestras 
aptitudes cognitivas a menudo, aunque tal vez no siempre, se asocian determinadas representaciones mentales que pueden ser contempladas como tokens de determinadas entidades abstractas. Sería posible, de hecho, mantener la posición de la pluralidad, y también su rechazo, del concepto de derecho en las tres concepciones. Sin embargo, tenerlas presentes nos hace más conscientes del trasfondo ontológico del debate.

En cambio, la segunda cuestión, la cuestión de la estructura de los conceptos es crucial para pronunciarnos sobre la plausibilidad de la pretendida pluralidad de conceptos de derecho.

Disponemos, en primer lugar, de la teoría clásica de los conceptos, de acuerdo con la cual un concepto es una entidad compuesta de otros conceptos más básicos que constituyen las condiciones necesarias y suficientes para determinar si determinados objetos, sucesos o individuos caen bajo la referencia de dicho concepto. Así, muchas veces desde Aristóteles se ha repetido que el concepto SER HUMANO está integrado por dos conceptos más básicos ANIMAL y RACIONAL. El problema más grave de la teoría es que pocas veces en filosofía las definiciones en términos de condiciones necesarias y suficientes han logrado su objetivo. El concepto SABER, como nos recuerdan Margolis y Laurence (2014), que desde Platón se suponía compuesto por los conceptos CREENCIA JUSTIFICADA VERDADERA, no ha podido superar el desafío de Gettier (1963) y la epistemología contemporánea puede ser vista, parcialmente, como un intento de enmendar o, al menos, completar esta definición. El concepto SABER ha devenido un concepto controvertido.

Por dicha razón, y otra vez de la mano de las ideas wittgenstenianas, se ha desarrollado la denominada teoría de los prototipos. Aquí ya no se exige una estructura definicional sino meramente una estructura probabilística: para que una mata que hay en mi jardín caiga bajo el concepto ARBUSTO basta que reúna un determinado y suficiente número de propiedades que no coinciden siempre con las propiedades que reúne otra mata que también cae bajo el concepto ARBUSTO. La idea wittgensteniana de parecido de familia ha sido desarrollada en psicología (véase. por ejemplo Rosch 1975).

Una concepción algo distinta es la que se halla en la teoría del enfoque teórico de los conceptos (the theory-theory of concepts). Según dicha concepción, no es posible analizar el núcleo de nuestros conceptos sin poner en relación unos conceptos con otros, igual como sucede con los términos teóricos de las teorías científicas. Moldear un concepto es elaborar una teoría lo suficientemente amplia que lo incluya para explicar una parte de la realidad. El contenido del concepto está dado por la función que cumple en la teoría. 
Hay también el denominado atomismo conceptual, con arreglo al cual los conceptos carecen de estructura semántica, su contenido no está determinado por su relación con otros conceptos, sino por su relación con el mundo. Es una idea que sigue la huella antidescriptivista de las teorías causales de la referencia (Kripke 1980, Putnam 1975) y que goza de algún predicamento en la filosofía actual (Fodor 1998). Así, el concepto ÁRBOL se refiere a una determinada composición genética vegetal que no depende de lo que los hablantes tenemos en nuestra cabeza (es una expresión que hizo fortuna con Putnam 1975) cuando usamos las palabras, sino de cómo los conceptos refieren al mundo.

Dado este panorama, algunos autores se han inclinado por sostener que los conceptos tienen múltiples estructuras (cf. Weiskopf 2009). Un concepto como ARBUSTO en realidad es una pluralidad de estructuras, representaciones mentales, prototipos, elementos de una teoría y, por lo tanto, no hay un concepto ARBUSTO, sino ARBUSTO ${ }_{1}, \mathrm{ARBUSTO}_{2}$, $\mathrm{ARBUSTO}_{3}$, etc. A algunos esta posición les conduce al eliminacionismo conceptual, a la supresión de los conceptos de nuestro arsenal filosófico puesto que no hay nada en común que nos permita introducir estas estructuras en nuestro arsenal filosófico (Machery 2009).

En varios lugares Dworkin (véase, sobre todo, 2006b, pp. 9-12) ha prestado atención a esta cuestión y ha sostenido que existen, al menos, tres tipos de conceptos: criteriológicos, de clase natural e interpretativos. Los primeros se corresponden con la teoría clásica de los conceptos, la teoría que establece condiciones necesarias y suficientes. Así, el concepto TRIÁNGULO EQUILÁTERO es aquel polígono de tres lados de igual longitud. Los conceptos de clase natural se corresponden con la teoría del atomismo conceptual que considera, por ejemplo, que el concepto AGUA se refiere a la estructura atómica $\mathrm{H}_{2} \mathrm{O}$, o el concepto ELEFANTE a determinada estructura genética, $\mathrm{ADN}$, de un animal. Dworkin, como es sabido, considera que el concepto DERECHO, como DEMOCRACIA, JUSTICIA, IGUALDAD y otros conceptos políticos, no son moldeables por las dos anteriores teorías. Son conceptos que presuponen una práctica, social y discursiva, pero en el seno de dicha práctica existe un amplio ámbito para la discrepancia y, por lo tanto, para la identificación de referentes distintos para distintos agentes. Creo que algo como el enfoque teórico de los conceptos es lo que Dworkin tiene en mente. Una interpretación, en estos ámbitos prácticos, funciona de manera semejante a como funciona una teoría científica.

Creo que Dworkin lleva razón en este punto. Pero creo que su concepción interpretativa debe ser completada por una teoría como la de los prototipos. Para que sepamos que nos estamos refiriendo al concepto 
DERECHO ESPAÑOL, para tener una pauta que unifique nuestras discrepancias, es preciso anclar dicho concepto en una práctica compartida. Las reflexiones de Dworkin (1986, pp. 65-66) acerca de la fase preinterpretativa, en donde se identifica el núcleo de las pautas relevantes, contiene una sugerencia de esta idea. Alguien que no reconociera el conjunto de normas de la Constitución española de 1978 como parte central del derecho español no estaría en condiciones de ofrecer una concepción adecuada y no estaría en posesión del concepto DERECHO ESPAÑOL.

Es cierto, sin embargo, que en alguna ocasión Dworkin desafía esta noción de prototipos o, como los denomina, casos paradigmáticos y sostiene (Dworkin 1986, p. 72) que:

Los paradigmas anclan las interpretaciones, pero ningún paradigma está seguro del desafío procedente de una nueva interpretación que da cuenta mejor de otros paradigmas y deja al anterior aislado como un error.

Puede ser que Dworkin lleve razón en algunos casos, pero como bien ha mostrado Endicott (1998), la afirmación contiene una exageración retórica. Igual que los conceptos de clases naturales tienen casos paradigmáticos (el pino que veo desde la ventana de mi jardín es un prototipo de árbol, por ejemplo) los productos sociales de carácter intencional tienen paradigmas. El concepto CANCIÓN está ejemplificado paradigmáticamente por Yesterday y el concepto NOVELA por La fiesta del Chivo de Mario Vargas Llosa. Alguien que disputa esto, no comprende el lenguaje, no está en posesión de los conceptos CANCIÓN y NOVELA.

Con lo cual, la que creo la mejor reconstrucción de la estructura del concepto DERECHO es aquella que combina la teoría de los prototipos con el enfoque teórico de los conceptos. Es decir, aquella que da cuenta de la capacidad de los sujetos cognitivos de discriminar casos paradigmáticos de derecho, que se manifiesta en circunstancias como aquellas en las que estando en un país extraño para nosotros preguntamos a nuestros anfitriones si el derecho del país prohíbe o no fumar en los restaurantes, y que moldea el concepto DERECHO para dar cuenta de su contenido en una tarea que tiene una dimensión social (que explica que algunos sistemas jurídicos prohíben fumar en los restaurantes y otros no lo prohíben), y una dimensión evaluativa, capaz de establecer en qué medida la existencia del derecho presupone determinados fundamentos morales, que permite relacionar el concepto DERECHO con otros conceptos como MORAL, JUSTICIA o COACCIÓN. 
En este punto, como puede apreciarse, mi acuerdo con las concepciones de Dworkin y Nino es muy amplio. También ellos creen relevante tomarse en serio la dimensión del derecho como práctica social y la dimensión del derecho como práctica justificativa de las acciones y de las decisiones institucionales. Lo único que critico de sus enfoques es que no apreciaran con claridad que para conseguir dicho fin, y anudar ambas dimensiones, es preciso consideran que están imbricadas en el mismo concepto. De no ser así, no estamos en condiciones de dar cuenta de la complejidad del fenómeno jurídico, apto a la vez para regular el comportamiento y estabilizar las expectativas de los miembros de nuestras sociedades y dar acomodo en su seno a genuinos y_profundos desacuerdos acerca de su contenido.

\section{Conclusión}

El concepto DERECHO, cuando equivale al concepto SISTEMA JURÍDICO, es un concepto con una estructura compleja. Moldearlo y estar en posesión de él presupone ser capaz de comprender su dimensión social y de capturar algunos rasgos sobresalientes que están presentes en los casos paradigmáticos de aplicación del concepto. Es una idea que subyace al esfuerzo de Hart de explicar la naturaleza del derecho. Los usuarios competentes del concepto DERECHO son capaces de comprender, como bien dice Hart (1961/2012, p. 3), que un sistema jurídico contiene los siguientes rasgos sobresalientes:

(i) reglas que prohíben o prescriben ciertos tipos de conducta bajo la amenaza de aplicar una pena; (ii) reglas que exigen que indemnicemos a quienes hemos dañado de cierta manera; (iii) reglas que especifican qué es lo que tenemos que hacer para otorgar testamentos y celebrar contratos $\mathrm{u}$ otros negocios jurídicos que confieren derechos y obligaciones; (iv) tribunales que determinan cuáles son las reglas y cuándo han sido vulneradas y fijan el castigo a aplicar o la compensación que debe satisfacerse; (v) una legislatura que crea nuevas reglas y deroga algunas de las anteriores.

Es cierto, también, que Hart no prestó seguramente la atención debida al hecho de que hay diversos modos de reconstruir los fundamentos que permiten establecer el contenido del derecho y que por dicha razón el concepto DERECHO es controvertido: hay diversas teorías del concepto y de la relación con otros conceptos que requieren embarcarse en una tarea evaluativa. En especial, de ahí la insistencia de Dworkin, 
en qué medida los fundamentos del contenido de aquello que es el derecho presuponen consideraciones y argumentos morales. ${ }^{11}$

El problema de la posición de Dworkin acerca de la pluralidad de los conceptos DERECHO tal vez resida en no haberse dado cuenta de que sin atender a la dimensión social del derecho no podemos elaborar el concepto doctrinal. ${ }^{12}$ Para distinguir el sistema fiscal español del francés es preciso atender a algún modo de distinguir y separar las prácticas jurídicas de creación de las normas sobre impuestos españolas y francesas; solo una vez que hemos logrado este fin es posible involucrarse en el debate doctrinal, por ejemplo si, conforme al derecho español, determinados pagos en especie suponen ingresos a los efectos del impuesto sobre la renta de las personas físicas. El contenido del derecho es la dimensión interna de una práctica social que habita en el mundo y genera relaciones causales en él, es un contenido intencional incorporado en determinadas prácticas institucionales.

El problema de la posición de Nino, en cambio, es tal vez no haber prestado la atención debida al hecho de que no puede haber un concepto DERECHO meramente descriptivo, por la razón esgrimida por Joseph $\operatorname{Raz}(2001$, p. 21):

Al fin y al cabo la propia comprensión de Hart de su teoría, este es el fin de la cuestión, rechaza que la explicación de la naturaleza del derecho sea evaluativa. Para él, se trataba de una tarea 'descriptiva'. Por razones explicadas por John Finnis ${ }^{13}$ y otros, creo que Hart está aquí en un error, y Dworkin está en lo cierto cuando sostiene que la explicación de la naturaleza del derecho involucra consideraciones evaluativas.

Por lo tanto, los conceptos DERECHO y SISTEMA JURÍDICO, son conceptos con una estructura compleja, porque se refieren a productos sociales de carácter intencional. Tienen una dimensión social, externa, que descansa en un conjunto de prototipos incorporados en nuestras prácticas sociales y que somos capaces de discriminar. Son estas prácticas las que nos hacen accesible su contenido significativo, su dimensión interna, a pesar de que los fundamentos que ancoran dicho contenido son controvertidos. Pero este hecho no ha de llevarnos a considerar que existen diversos conceptos de derecho, sino a seguir argumentando para desarrollar la mejor teoría que dé cuenta de ambas dimensiones. Suponer

11 Véase el lúcido e influyente trabajo de Greenberg (2004) al respecto.

12 Aunque pueden verse sus consideraciones en el último capítulo de Dworkin (2011).

${ }^{13}$ Cf. Finnis (1980, cap. 1). 
que estas controversias y desacuerdos muestran que estamos frente a debates de palabras es, como mínimo, una asunción carente de la suficiente justificación. Son controversias genuinas y solo tomándolas de este modo conseguiremos agudizar nuestra propia concepción de la naturaleza del derecho.

Parte de ello anima, según creo, la crítica que Genaro R. Carrió (1990) realizó a esta tesis de Nino. ${ }^{14}$ Según Carrió, también el positivismo jurídico trata de dar cuenta de la dimensión normativa del derecho y Nino, según Carrió (1990, p. 401), presenta de modo sesgado las posiciones iuspositivistas como si solo reconstruyeran la dimensión descriptiva del derecho. No creo, sin embargo, que la reconstrucción de la dimensión normativa del derecho por parte de Carrió esté exenta de problemas y, en este punto, Nino presentaba dicha dimensión de un modo más perspicuo. Ahora bien, en relación con la tesis de la pluralidad de conceptos de derecho, insisto, creo que Carrió llevaba la razón y que la polémica sobre la naturaleza del derecho no se puede despachar como una mera cuestión de palabras. Como otros conceptos con dimensión normativa, la naturaleza de lo que representan es controvertida. Como una vez dijera von Wright (1963, p. 6), las palabras (como 'derecho', 'democracia', 'justicia', por ejemplo) "están, por así decirlo, a la búsqueda de un significado". ${ }^{15}$

\section{Bibliografía}

Alchourrón, C. E. y Bulygin, E. (1971), Normative Systems, Nueva York, Springer.

(1981), "The Expressive Conception of Norms" en Hilpinen, R. (ed.), New Studies in Deontic Logic, Dordrecht, Reidel, pp. 95-121.

Bayón, J. C. (1996), "Pertecipanti, osservatori, e identificazione del diritto",

${ }^{14}$ Carrió escribió su trabajo para un homenaje a Norberto Bobbio, publicado en 1983 (ahora en Carrió 1990) precisamente como objeción a un trabajo de Nino (donde tempranamente expone su tesis, Nino 1980, ahora en Nino 1985a) que era una crítica a Dworkin. Nino también replica a Carrió (Nino 1985 c), pero más interesado en defender su posición normativa que su tesis de la pluralidad de los conceptos de derecho. Una buena guía para orientarse en este laberinto es Roca (2005, pp. 138-161). También Ruiz Miguel (1997) cree, con buenas razones, que Nino no consigue mostrar que la polémica entre iusnaturalismo y iuspositivismo sea una mera cuestión de palabras. Sobre los argumentos de Ruiz Miguel puede verse también Bayón (1996) que distingue entre el derecho identificado desde el punto de vista del observador y el derecho identificado desde el punto de vista del participante. El interés de Bayón es epistémico más que conceptual y, por ello, sus argumentos no son analizados aquí.

${ }^{15}$ Deseo agradecer los valiosos comentarios que hicieron a una versión previa del trabajo Sebastián Agüero y un anónimo evaluador de Análisis Filosófico. 
en Commanducci, P. y Guastini, R. (eds.), Struttura e dinámica dei sistema giuridici, Turín, Giapichelli, pp. 47-64.

Carrió, G. R. (1983), "Una reciente propuesta de conciliación entre el jusnaturalismo y el positivismo jurídico", en Scarpelli, U. (ed.), La teoria generale del diritto: Problemi e tendenze attuali: Studi dedicati a Norberto Bobbio, Milán, Ed. di Comunità, pp. 361-385.

- (1990), "Un intento de superación de la controversia entre positivistas y jusnaturalistas (Réplica a Carlos S. Nino)", en Notas sobre derecho y lenguaje, Buenos Aires, Abeledo-Perrot, pp. 375-404.

Celano, B. (ms), Pre-convenzione: un frammento dello Sfondo.

Coleman, J. L. (1998), "Incorporationism, Conventionality, and the Practical Difference Thesis", Legal Theory, 4, pp. 381-426.

Dworkin, R. (1977), Taking Rights Seriously, Londres, Duckworth. (1986), Law's Empire, Cambridge, Harvard University Press. (2006a), "Hart and the Concepts of Law", Harvard Law Review Forum, 119, pp. 95-104.

(2006b), Justice in Robes, Cambridge, Harvard University Press. (2011), Justice for Hedgehogs, Cambridge, Harvard University Press.

Endicott, T. A. O. (1998), "Herbert Hart and the Semantic Sting", Legal Theory, 4, pp. 283-301.

Finnis, J. (1980), Natural Law and Natural Rights, Oxford, Oxford University Press.

Fodor, J. (1998), Concepts: Where Cognitive Science Went Wrong, Oxford, Oxford University Press.

Gardner, J. (2012a), "Law in General", en Law as a Leap of Faith, Oxford, Oxford University Press, cap.11.

(2012b), "The Legality of Law" en Law as a Leap of Faith, Oxford, Oxford University Press, cap. 7.

Gettier, E. L. (1963), "Is Justified True Belief Knowledge?", Analysis, 23, pp.121-123.

Greenberg, M. (2004), "How Facts Make Law”, Legal Theory, 10, pp.157-198. Hart, H. L. A. (1961/2012), The Concept of Law, Oxford, Oxford University Press, $3^{\mathrm{a}}$ ed. con una introducción de Leslie Green.

Kelsen, H. (1960), Reine Recthslehre, Viena, Franz Deuticke, $2^{\mathrm{a}}$ ed.

Kripke, S. (1980), Naming and Necessity, Oxford, Basil Blackwell.

Laporta, F. J. (2007), El imperio de la ley: Una visión actual, Madrid, Trotta.

Machery, E. (2009), Doing without Concepts, Oxford, Oxford University Press.

Margolis, E. y Laurence, S. (2014), “Concepts”, en Zalta, E. N. (ed.), The 
Stanford Encyclopedia of Philosophy (Spring 2014 Edition). Disponible en http://plato.stanford.edu/archives/spr2014/entries/concepts/.

Moreso, J. J. (2009), La Constitución: modelo para armar, Madrid, Marcial Pons.

(2013), Ethica more iuridico incorporata, México, Fontamara.

Nino, C. S. (1980), "Dworkin and Legal Positivism", Mind, 89, pp. 519-543. (1985a), "La superación de la controversia "positivismo vs. iusnaturalismo' a partir de la ofensiva antipositivista de Dworkin", en La validez del derecho, Buenos Aires, Astrea, pp. 145-174.

_ (1985b), "El enfoque esencialista del concepto de derecho" en La validez del derecho, Buenos Aires, Astrea, pp.175-196.

- (1985c), "Hay deberes y 'deberes': respuesta a Carrió”, en La validez del derecho, Buenos Aires, Astrea, pp. 209-222.

- (1994), Derecho, moral y política: Una revisión de la teoría general del derecho, Barcelona, Ariel.

Peacocke, C. (1992), A Study of Concepts, Cambridge, The MIT Press.

Pinker, S. (1994), The Language Instinct: The New Science of Language and Mind, Londres, Penguin.

Putnam, H. (1975), "The Meaning of 'Meaning" en Mind, Language and Reality, Cambridge, Cambridge University Press, pp. 215-271.

Raz, J. (1979), The Authority of Law, Oxford, Oxford University Press.

- (1994a), "Authority, Law, and Morality" en Ethics in the Public Domain, Oxford, Oxford University Press, cap. 10.

(1994b), 'The Problem about the Nature of Law', en Ethics in the Public Domain, Oxford, Oxford University Press, cap. 9.

(2001), "Two Views of the Nature of the Theory of Law: A Partial Comparison", en Coleman, J. (ed.), Hart's Postscript, Oxford, Oxford University Press, cap. 1.

(2004), "Incorporation by Law", Legal Theory, 10, pp. 1-17.

Roca, V. (2005), Derecho y razonamiento práctico en Carlos S. Nino, Madrid, Centro de Estudios Políticos y Constitucionales.

Rosch, E. (1975), "Cognitive Representation of Semantic Categories", Journal of Experimental Psychology, 104, pp. 192-233.

Ross, A. (1958), On Law and Justice, Londres, Stevens \& Sons.

Ruiz Miguel, A. (1997), "Derecho y punto de vista moral", Anuario de Filosofía del Derecho, 14, pp. 571-597.

Searle, J. (1983), Intentionality: An Essay in the Philosophy of Mind, Cambridge, Cambridge University Press.

Shapiro, S. J. (2011), Legality, Cambridge, Harvard University Press. Von Wright, G. H. (1963), The Varieties of Goodness, Londres, Routledge \& Kegan Paul. 
Weiskopf, D. A. (2009), "The Plurality of Concepts”, Synthese, 169, pp. 145-173.

Wittgenstein, L. (1953), Philosophical Investigations, editado por G. E. M. Anscombe, Oxford, Basil Blackwell.

Recibido el 1 de septiembre de 2014; aceptado el 14 de noviembre de 2014. 